\title{
Décision de la Commission d'interprétation paritaire
}

TARMED Suisse

\section{Interprétation}

Principe de base: La réglementation transitoire n'est applicable que si

- les prestations SSO sont fournies sous anesthésie en salle d'opération, ou que

- les prestations SSO sont fournies en combinaison avec des prestations TARMED sous anesthésie en salle d'opération.

Si la facturation ne concerne que des prestations TARMED, seules les dispositions dudit tarif sont applicables.

A) Facturation de prestations SSO sous anesthésie en salle d'opération

1. Les prestations de médecine dentaire sont facturées selon les positions du tarif SSO.

2. Les prestations d'anesthésie sont facturées selon les positions tarifaires du TARMED et en fonction des classes de risque mentionnées en annexe:

Si plusieurs prestations comportant diverses classes de risque anesthésique sont fournies au cours d'une même opération, c'est la classe de risque de la prestation présentant la classe de risque anesthésique la plus élevée qui est applicable pour les positions «prise en charge périopératoire», «induction et réveil» ainsi qu'«activité du médecin anesthésiste pendant l'intervention».

En principe, la classe de risque anesthésique III est applicable pour les interventions sur des enfants de moins de deux ans.

Pour le temps d'anesthésie facturable durant l'intervention (28.0140, 28.0150), c'est la durée effective de l'anesthésie qui compte (durée incision-suture) conformément au protocole d'anesthésie. Le protocole d'anesthésie doit être remis immédiatement et gratuitement au répondant des frais avec mention du numéro de la facture, sans que ce dernier en fasse la demande.

3. Le matériel à usage courant et les anesthésiques utilisés pour les prestations d'anesthésie sont facturables en plus selon les interprétations IG-20 et IC-28-3.

4. L'assistance médicale est facturée selon les chiffres 4980 ou 4981 du tarif SSO.

5. L'indemnisation pour l'utilisation de la salle d'opération se fait selon le chiffre $4983 \mathrm{du}$ tarif SSO.

6. L'indemnisation du lit et de la surveillance a lieu selon les chiffres 4985 et 4986 du tarif SSO.

7. Pour la facturation du matériel à usage courant, les dispositions du tarif SSO, chiffre 4983, sont applicables.

B) Facturation de prestations SSO en combinaison avec des prestations TARMED sous anesthésie en salle d'opération

1. Les prestations de médecine dentaire sont facturées selon les positions du tarif SSO.

2. Les prestations TARMED sont facturées selon les positions tarifaires du TARMED.

3. Lorsque des prestations ou des prestations partielles figurent simultanément dans le tarif de la SSO et dans le TARMED, il convient de choisir la variante la plus économique.

4. Les prestations d'anesthésie sont facturées selon les positions tarifaires du TARMED, et ce en fonction des classes de risque correspondantes mentionnées en annexe (pour les prestations SSO) et dans le TARMED (pour les prestations TARMED):

Si plusieurs prestations comportant diverses classes de risque anesthésique sont fournies au cours d'une même opération, c'est la classe de risque de la prestation présentant la classe de risque anesthésique la plus élevée qui est applicable pour les positions «prise en charge périopératoire», «induction et réveil» ainsi qu' «activité du médecin anesthésiste pendant l'intervention».

En principe, la classe de risque anesthésique III est applicable pour les interventions sur des enfants de moins de deux ans. 
Pour le temps d'anesthésie facturable durant l'intervention $(28.0140,28.0150)$, c'est la durée effective de l'anesthésie qui compte (durée incision-suture) conformément au protocole d'anesthésie. Le protocole d'anesthésie doit être remis immédiatement et gratuitement au répondant des frais avec mention du numéro de la facture sans que ce dernier en fasse la demande.

5. Le matériel à usage courant et les anesthésiques utilisés pour les prestations d'anesthésie sont facturables en plus selon les interprétations IG-20 et IC-28-3.

6. La prise en charge non médicale est facturée selon les positions tarifaires du chapitre 35.03 du TARMED.

7. Dans les positions tarifaires TARMED, l'assistance médicale fait partie intégrante des prestations concernées et est indemnisée par le biais de celles-ci. L'assistance médicale pour les prestations dentaires du tarif SSO est indemnisée au moyen des chiffres 4980 ou 4981 du tarif SSO.

8. L'indemnisation pour l'utilisation de la salle d'opération se fait selon le chiffre $4983 \mathrm{du}$ tarif SSO. La prestation de base technique «salle d'opération» du TARMED (chapitre 35.01) peut-être facturée en plus, tout en tenant compte de la remarque concernant les interventions multiples qui figure dans l'interprétation IC-35.01-1.

9. Pour la facturation du matériel à usage courant dans le cadre des positions du tarif SSO, les dispositions du tarif SSO, chiffre 4983, sont applicables. Pour la facturation du matériel à usage courant en combinaison avec des positions tarifaires TARMED, les dispositions de l'interprétation IG-20 du TARMED sont applicables. Le matériel à usage courant indemnisé via le chiffre 4983 du tarif SSO conformément aux explications figurant sous ledit chiffre, ne peut pas être facturé une nouvelle fois de façon séparée en vertu de l'interprétation IG-20 du TARMED.

\section{Annexe}

A) Explications concernant la facturation de la classe de risque anesthésique III pour les prestations de médecine dentaire et les interventions de chirurgie maxillofaciale facturées selon le tarif SSO

Dans les cas mentionnés ci-après, la classe de risque anesthésique III est applicable (de façon sélective) pour la totalité de la durée incisionsuture (selon le protocole d'anesthésie):
- pour les prestations des chapitres V ou VI comportant une classe de risque anesthésique III (cf. section B ci-après);

- pour les enfants de moins de 2 ans;

- lorsque le protocole d'anesthésie indique que le patient n'a pas pu coopérer en raison de son état mental, psychique ou corporel;

- lorsque le protocole d'anesthésie mentionne que le patient appartient à la classe ASA 3 ou à une classe plus élevée.

Pour tous les autres cas que ceux mentionnés cidessus, la classe de risque anesthésique II peut être facturée.

Les classes de risque anesthésique I et IV ne sont pas facturées.

\section{B) Classe de risque anesthésique du tarif SSO (tarif dentaire), chapitre V et VI}

\begin{tabular}{ll}
$\begin{array}{ll}\text { Chapitre V: Chirurgie dentaire, chirurgie orale } \\
\text { Position du tarif SSO }\end{array}$ & $\begin{array}{l}\text { Classe de risque } \\
\text { anesthésique }\end{array}$ \\
\hline $4200-4211$ & III \\
4212 & II \\
$4213-4224$ & III \\
$4224-4226$ & II \\
\hline $4227-4248$ & III \\
\hline 4250 & aucune \\
\hline $4251-4269$ & III \\
\hline $4270-4271$ & II \\
\hline $4272-4282$ & III \\
\hline $4283-4284$ & II \\
\hline $4285-4286$ & III \\
\hline 4287 & II \\
\hline 4288 & III \\
\hline 4290 & II \\
\hline $4291-95$ & III \\
\hline $4296-4299$ & III \\
\hline
\end{tabular}

\begin{tabular}{|c|c|}
\hline \multicolumn{2}{|c|}{ Chapitre VI: Chirurgie maxillo-faciale } \\
\hline Position du tarif SSO & $\begin{array}{l}\text { Classe de risque } \\
\text { anesthésique }\end{array}$ \\
\hline $4300-4325$ & III \\
\hline $4326-4328$ & aucune \\
\hline $4330-4334$ & II \\
\hline $4335-4337$ & III \\
\hline $4340-4342$ & II \\
\hline $4344-4355$ & III \\
\hline 4356 & aucune \\
\hline $4357-4361$ & III \\
\hline $4363-4365$ & II \\
\hline 4366 & III \\
\hline $4367-4368$ & II \\
\hline $4370-4394$ & III \\
\hline
\end{tabular}

